Research Paper

\title{
The caudal pedunculopontine tegmental nucleus may be involved in the regulation of skeletal muscle activity by melanocortin- sympathetic pathway: a virally mediated trans-synaptic tracing study in spinally transected transgenic mice
}

\author{
Zhi-Gang Heㄹ, Bao-Wen Liư ${ }^{1}$, Zhi-Xiao Li' ${ }^{1}$, Xue-bi Tian ${ }^{1}$, San-Guang Liu ${ }^{2}$, Anne \\ Manyande ${ }^{3}$, Ding-Yu Zhang ${ }^{4}$ and Hong-Bing Xiang ${ }^{1}$ \\ ${ }^{1}$ Department of Anesthesiology and Pain Medicine, Tongji Hospital of Tongji Medical College, Huazhong University of Science \\ and Technology, Wuhan, Hubei, People's Republic of China \\ ${ }^{2}$ Department of Hepatobiliary Surgery, The Second Hospital, Hebei Medical University, Shijiazhuang, People's Republic of \\ China \\ ${ }^{3}$ School of Human and Social Sciences, University of West London, London, UK \\ ${ }^{4}$ Intensive Care Unit, Wuhan Medical Treatment Center, Wuhan, P. R. China \\ Correspondence to: Hong-Bing Xiang, email: hbxiang@tjh.tjmu.com.cn
}

Keywords: pedunculopontine tegmental nucleus, melanocortin-4 receptor, skeletal muscle, pseudorabies virus, mouse

Received: October 04, 2016

Accepted: March 28, 2017

Published: May 18, 2017

Copyright: He et al. This is an open-access article distributed under the terms of the Creative Commons Attribution License 3.0 (CC BY 3.0), which permits unrestricted use, distribution, and reproduction in any medium, provided the original author and source are credited.

\section{ABSTRACT}

Understanding neuroanatomical sympathetic circuitry and neuronal connections from the caudal pedunculopontine tegmental nucleus to skeletal muscle is important to the study of possible mechanisms of pedunculopontine tegmental nucleus (PPTg) and cuneiform nucleus ( $\mathrm{CnF}$ ) that are involved in the regulation of skeletal muscle activity of the sympathetic pathway. The aim of this study was to use virus PRV-614 to trace the melanocortin-sympathetic neural pathways from PPTg and $\mathrm{CnF}$ to a hindlimb muscle (gastrocnemius) in spinally transected MC4R-GFP transgenic mice. PRV-614 was injected into the gastrocnemius muscle after receiving a complete spinal cord transection below the L2 level. PRV-614/MC4R-GFP and PRV-614/TPH dual-labeled neurons were found in the dissipated parts of PPTg (dpPPTg), but not between the compact parts of PPTg (cPPPTg) and CnF. It is proposed that a hierarchical pathway of neurons within the caudal pedunculopontine tegmental nucleus sends projections to the RVLM, which in turn projects onto the IML sympathetic preganglionic neurons that regulate muscle blood flow through melanocortin-sympathetic signals. Our results collectively indicate that MC4Rs expressed in caudal pedunculopontine tegmental nucleus may be involved in skeletal muscle activity of melanocortin-sympathetic pathways.

\section{INTRODUCTION}

Much evidence supports the claim that cholinergic neuronal loss within the mesencephalic pedunculopontine tegmental nucleus (PPTg) is associated with the level of dopaminergic degeneration [1] and the clinical severity of the characteristic motor symptoms of Parkinson's disease: postural instability and gait disturbances [2, 3]. PPTg deep brain stimulation (DBS) used to treat these symptoms has also provided some insight into the function of PPTg [4-6]. Studies that evaluated the safety and efficacy of PPTg DBS, failed to establish the optimal site and precise effects of PPTg DBS of certain motor symptoms of Parkinson's disease. In an earlier study, we reported the existence of a direct neuronal circuit from the caudal pedunculopontine tegmental nucleus and cuneiform nucleus $(\mathrm{CnF})$ to the skeletal muscle through the motor pathway [7]. It is however, unclear whether these effects of PPTg DBS are partially mediated through the sympathetic connections of this neuronal circuit. Clearly, it is important to study the possible mechanism of pedunculopontine tegmental nucleus and cuneiform nucleus involved in the regulation of skeletal muscle activity of the sympathetic pathway. 
There are reports that the pedunculopontine tegmental nucleus which coexpresses choline acetyltransferase (a marker of cholinergic neurons) immunoreactivity and melanocortin-4 receptor (MC4R) expression [8, 9], is involved in mechanisms of behavioral state control, locomotion and muscle tone control $[7,10,11]$. In addition, the central melanocortin system has also been found to be a critical mediator of skeletal muscle activity [12]. Other studies have shown that central regulation of sympathetic nerve activity is a major component of melanocortinergic action [8, 13, 14] and that central serotonergic and catecholaminergic positive neurons regulate sympathetic outflow $[15,16]$. More recently, MC4Rs expressed by cholinergic neurons (including sympathetic preganglionic motor neurons) [17] in the central nervous system were shown to be key regulators of energy and glucose homeostasis through the activity of the autonomic nervous system [18-27]. We therefore, suggest that the mesencephalic locomotor regions are closely linked to skeletal muscle activity through melanocortin-sympathetic signals.

In yet other studies, the neurotropic tracer microinjections are reported to be very widely used in brain mapping research [7, 15, 28-39]. The transneuronal tracer pseudorabies virus (PRV) was used to investigate whether connections from the gastrocnemius muscle to the mesencephalic locomotor region (MLR), which is composed of the pedunculopontine nucleus (PPTg) and the adjacent cuneiform nucleus ( $\mathrm{CnF}$ ) [40], are maintained by largely separate populations of neurons. The retrograde tracer PRV-614, was injected into the gastrocnemius muscle of MC4R-green fluorescent protein (GFP) transgenic mice after receiving a complete spinal cord transection below the L2 level using an electrocautery $[16,41,42]$. The aim of the present study was therefore, to use PRV-614 in order to trace the melanocortin-sympathetic neural pathways from pedunculopontine tegmental nucleus and cuneiform nucleus to the gastrocnemius muscle in spinally transected transgenic mice.

\section{RESULTS}

\section{Specific expression of PRV-614 in the pedunculopontine nucleus areas}

Animals received a successful transection in the L2 spinal cord, as indicated by the lack of neurons immunopositive for PRV-614 in the ventral horn (VH) of the spinal cord. At $5 \mathrm{~d}$ after PRV-614 injection into the gastrocnemius muscle, retrograde infection of neurons were noticed in the spinal cord, medulla oblongata, and pedunculopontine nucleus areas. PRV-614-labeled neurons were found in the IML, RVLM, RVMM, and the dpPPTg but not in the $\mathrm{CnF}$ and cpPPTg (Figure 1A).

\section{MC4R and PRV-614 co-expression in the pedunculopontine nucleus areas}

We examined GFP expression in the MC4RGFP reporter mouse and observed a large number of GFP-positive neurons in the IML, medulla, and pedunculopontine nucleus areas. PRV-614/MC4R-GFP and PRV-614/TPH dual labeled neurons were found in the dpPPTg (Figure 1D, 1E and not in the $\mathrm{CnF}$ and cpPPTg (Figure 1E) after complete spinal cord transection. Duallabeled PRV-614/MC4R-GFP neurons were coexpressed in the RVLM, RVMM, and IML (Figure 2, yellow, some are indicated by white arrows) but not in the VH (Figure 2). The percentage of PRV-614-colocalization in MC4R-GFPpositive neurons of the dpPPTg was $26.7 \%-30 \%$, whereas that of PRV-614-colocalization in TPH-positive neurons of the dpPPTg was 20\%-30\%.

\section{DISCUSSION}

In the present study, we utilized PRV-614 and MC4R-GFP mouse model to demonstrate that in spinally transected transgenic mice, the caudal pedunculopontine tegmental nucleus may be involved in the regulation of skeletal muscle activity of the melanocortin-sympathetic pathway. The principal findings of this study were: (1) specific midbrain regions, particularly the caudal pedunculopontine tegmental nucleus (Figure 1A), contained a substantial number of neurons that were infected 5 days following injections of PRV-614 into the gastrocnemius muscle. (2) PRV-614-labeled neurons were found in the IML, RVLM, RVMM, but not in the CnF and cpPPTg. (3) PRV-614/MC4R-GFP and PRV-614/TPH dual labeled neurons were detected in the dpPPTg after a complete spinal cord transection.

Considering that the parasympathetic nervous system failed to provide any innervations to limb muscles [16] and the spinal cord was transected rostral to the gastrocnemius motoneuron pool, we could speculate that the caudal pedunculopontine tegmental nucleus neurons were infected with PRV-614 via the sympathetic nervous system. Another explanation could be that there existed a direct neuronal circuit from the caudal pedunculopontine tegmental nucleus to skeletal muscle via the sympathetic pathway after the spinal cord transection. It is also evident from the graphs in Figure 1 that very few viral-infected cells were detect in the $\mathrm{CnF}$ (Figure 1A). Alternatively, it may reflect that the $\mathrm{CnF}$ does not play a major role in the regulation of skeletal muscle tone of the sympathetic pathway, which was in agreement with a previous report [43].

Interestingly, graphs in Figure 1A indicate that relatively small numbers of MC4R-GFP-labeled cells were scattered in the $\mathrm{CnF}$ whereas the caudal pedunculopontine tegmental nucleus showed moderate to high levels of 
MC4R-GFP immunoreactivity. Previous investigations have reported the coexpression of ChAT immunoreactivity and MC4R expression in the peduncular pontine tegmental nucleus [9]. Based on these data, we can speculate that ChAT immunoreactivity mainly exists in the caudal pedunculopontine tegmental nucleus rather than in the $\mathrm{CnF}$. Other studies have found similar results in which the cholinergic neurons were preferentially distributed in the area corresponding to the inhibitory region (PPTg) rather than the locomotor region $(\mathrm{CnF})[44]$.

A further feature of the results confirms that PRV-614/ MC4R-GFP and PRV-614/TPH double-labeled neurons were observed in the dissipated parts of pedunculopontine tegmental nucleus (dpPPTg) (Figure 1D, 1E), but not in cpPPTg and $\mathrm{CnF}$. On this basis, we could further speculate that $\mathrm{CnF}$ and cpPPTg neurons do not regulate skeletal muscle activity via the melanocortin-sympathetic pathway unlike dpPPTg neurons. In essence, these data indicate that projections from the caudal pedunculopontine tegmental nucleus (dpPPTg and cpPPTg) and cuneiform nucleus to skeletal muscle arise from largely separate populations of neurons. Therefore, other interpretations should be considered, particular that deep brain stimulation of the caudal pedunculopontine tegmental nucleus could be involved in melanocortin-sympathetic, serotonergic and motor pathways.

Moreover, we found that PRV-614/MC4R-GFP neurons were in several portions of the medulla oblongata including the rostral ventrolateral, ventromedial medulla (RVLM and RVMM) (Figure 2A), and the spinal cord (Figure 2B), which were consistent with previous reports [45] This suggests that there are direct connections between RVLM premotor neurons and sympathetic preganglionic neurons located in the IML.

In conclusion, based on previous studies and our results, we can speculate that a hierarchical pathway has neurons within the caudal pedunculopontine tegmental nucleus that send projections to the RVLM, which in turn project to sympathetic preganglionic neurons within the IML, that regulate muscle blood flow through melanocortin-sympathetic signals (Figures 2 and 3). In addition, MC4Rs expressed in $\mathrm{CnF}$ and cpPPTg neurons do not regulate skeletal muscle tone via melanocortinsympathetic pathways unlike MC4Rs in dpPPTg. Further research will be necessary to test the validity of our findings.

\section{MATERIALS AND METHODS}

\section{Animals care}

Experiments were carried out on transgenic MC4RGFP mice, which were genotyped as described by Rossi and colleagues [20]. Mice were kept under controlled conditions $\left(24 \pm 0.5^{\circ} \mathrm{C}, 12 \mathrm{~h}\right.$ alternating light-dark cycle, food and water ad libitum). Experimental procedures and
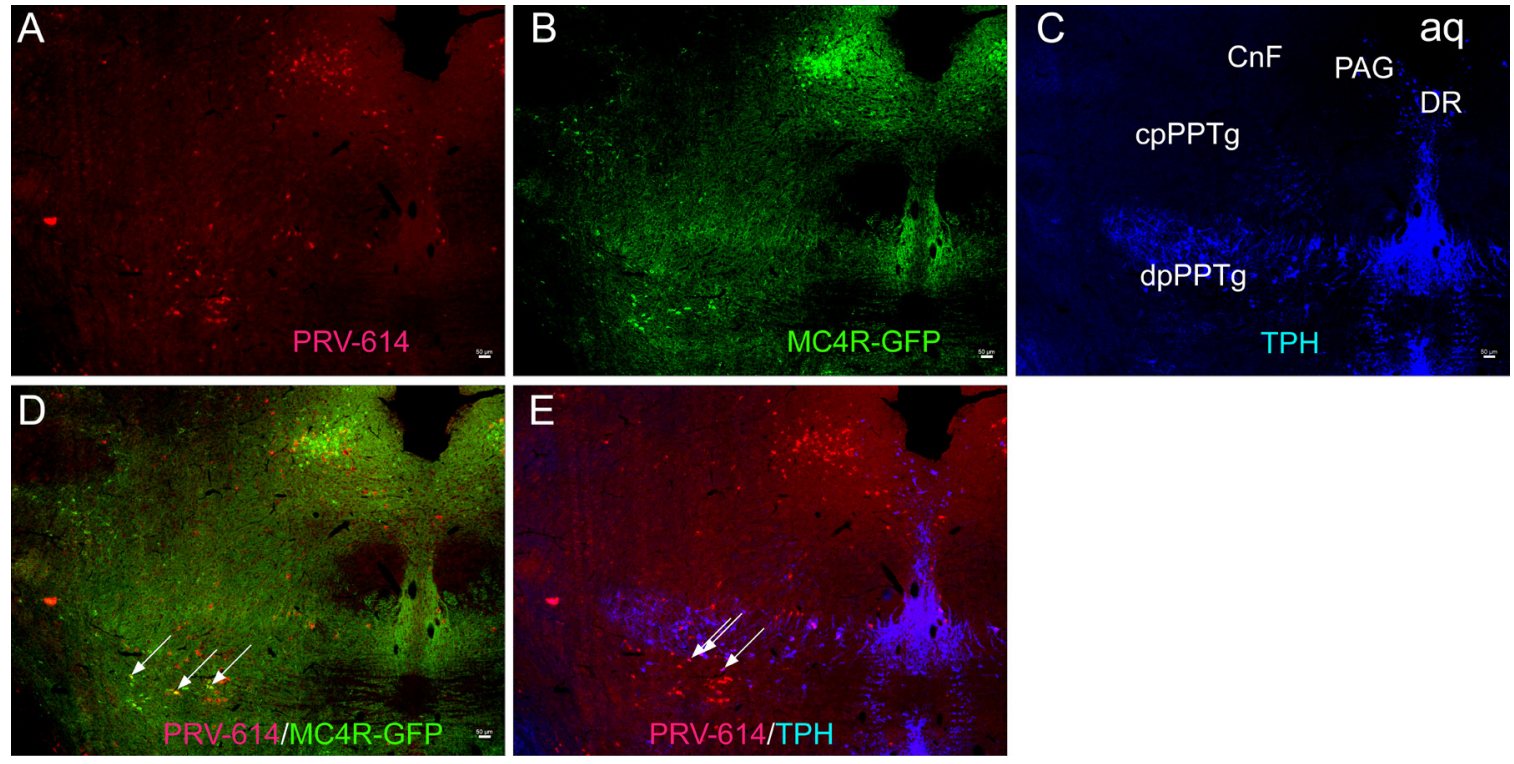

Figure 1: Micrographs illustrating sections through the $\mathrm{CnF}$ and caudal levels of the edunculopontine tegmental nucleus (PPTg). (A) Sections immunoreacted for PRV-614 (red). (B) Sections immunoreacted for MC4R-GFP (green). (C) Sections immunoreacted for TPH (blue). (D) Sections immunoreacted for PRV-614/MC4R-GFP dual labeled neurons (yellow, some are indicated by white arrows). (E) Sections immunoreacted for PRV-614/TPH dual labeled neurons (pink, some are indicated by white arrows). After a complete spinal cord transection, injections of PRV-614 into the gastrocnemius muscle resulted in retrograde infection of neurons in the CnF, cpPPTg and dpPPTg, and PRV-614/MC4R-GFP and PRV-614/TPH dual labeled neurons were detected in the dpPPTg. In contrast to the dpPPTg, we didn't detect dual labeled neurons in the CnF and cpPPTg. PRV-614, pseudorabies virus-614; CnF, cuneiform nucleus; Aq, aqueduct; DR, dorsal raphe; dpPPTg, the dissipated parts of PPTg; cpPPTg, the compact parts of PPTg; TPH, tryptophan hydroxylase. Scale bar: $50 \mu \mathrm{m}$. 
protocols used in this study were approved in advance by the Animal Care and Use Committee of Tongji hospital, Huazhong University of Science \& Technology (No. TJ-A20150805). This study also adhered to the guidelines of the U.K. Animals (Scientific Procedures) Act, 1986 and associated guidelines, the European Communities Council Directive of 24 November 1986 (86/609/EEC).
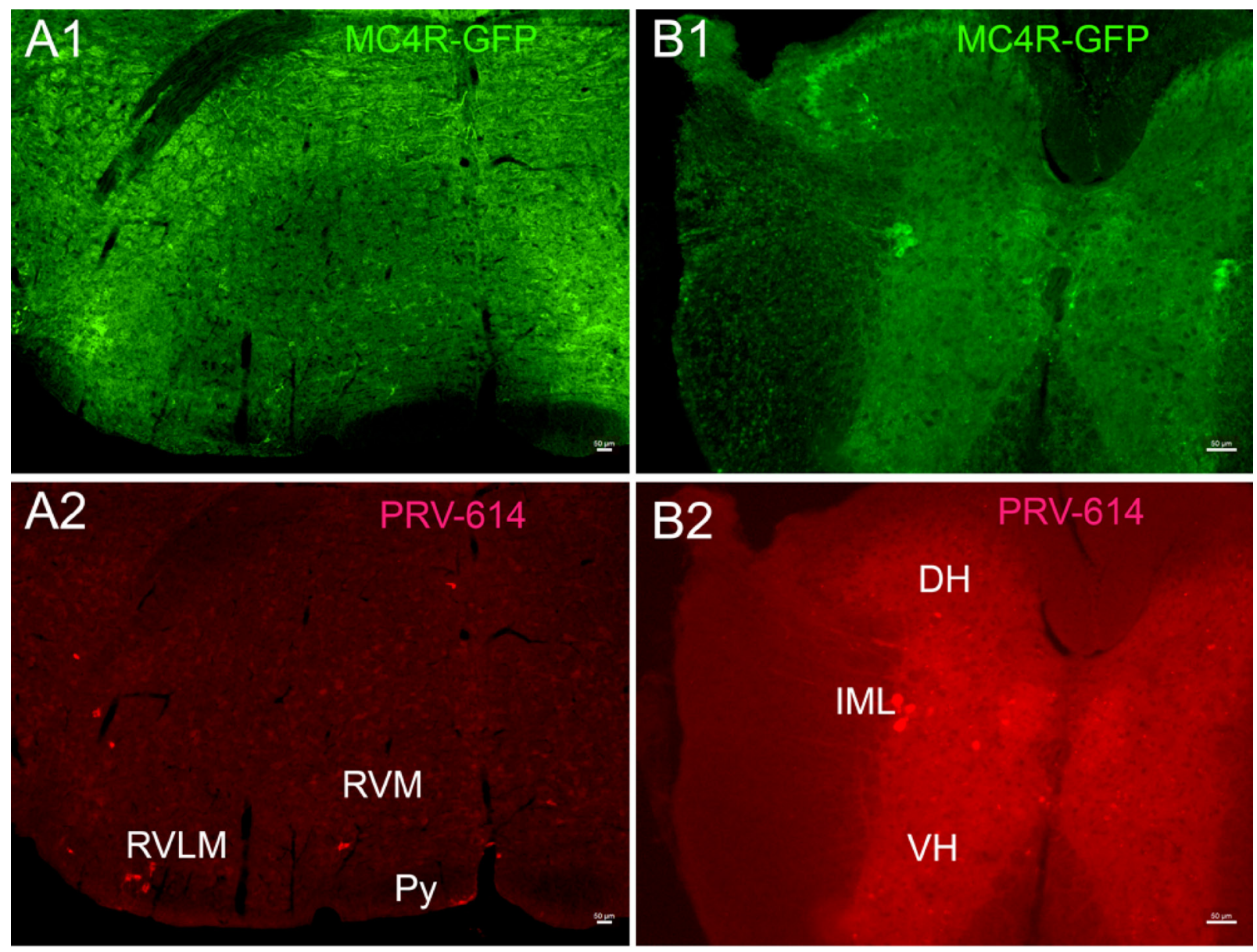

PRV-614

\section{$\mathrm{DH}$}
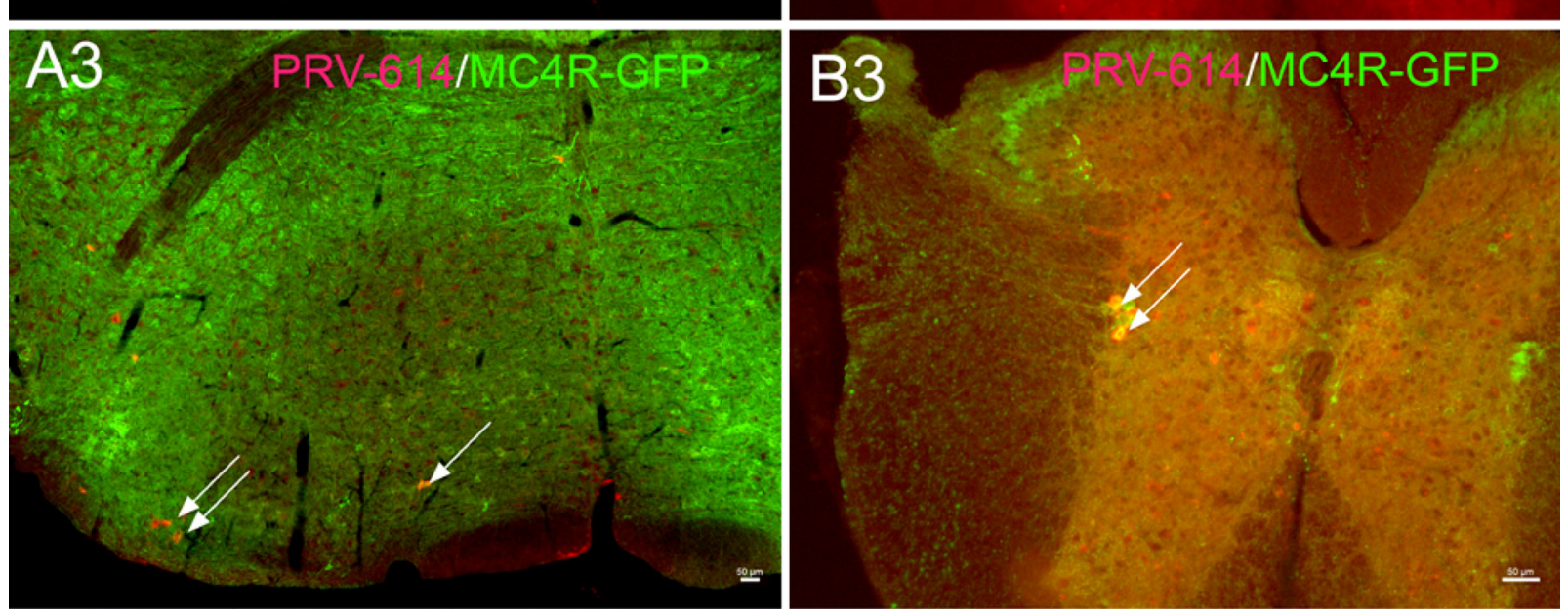

Figure 2: Gastrocnemius muscle cell groups target PRV-614 positive neurons (red) of the rostral ventral medulla (A) and spinal cord (B) levels. (A1 and B1) Sections immunoreacted for MC4R-GFP (green). (A2 and B2) Sections immunoreacted for PRV-614 (red). (A3 and B3) Sections immunoreacted for PRV-614/MC4R-GFP dual labeled neurons (yellow, some are indicated by white arrows). After a complete spinal cord transection, injections of PRV-614 into the gastrocnemius muscle resulted in retrograde infection of neurons in the RVLM, RVMM, and IML. In contrast to the IML, we didn't detect labeled neurons in the VH in spinally transected transgenic mice. PRV614, pseudorabies virus-614; IML, intermediolateral column; Py, pyramidal tract; RVLM, rostral ventrolateral medulla; RVMM, rostral ventromedial medulla; VH, ventral horn. Scale bar: $50 \mu \mathrm{m}$. 


\section{Spinal transection}

Male transgenic MC4R-GFP mice, weighing 25-30 g, were used in the experiments. The L2 spinal transection was performed in a manner similar to that described in previous reports [16]. Mice were provided analgesia based on a mixture of ketamine and ketoprofen. Following spinal transection, animals had their lower body paralyzed, and were maintained in a special cage so as to have access to food and water [16]. Gentle massage of the abdomen was operated to aid urination every $6 \mathrm{~h}[16]$.

\section{Microinjection of virus into the gastrocnemius muscle}

PRV-614 was microinjected into the gastrocnemius muscle at day 3 after spinal cord transection on male transgenic MC4R-GFP mice in a manner similar to previous reports [16]. In brief, $2 \mu 1$ injections of PRV-614 were inserted into the gastrocnemius muscle $(0.5 \mu \mathrm{l}$ per injection at 4 injection sites per one mouse). After every injection, the needle was kept in situ for 2 minutes to limit the spread of PRV-614.

\section{Perfusion and fluorescence immunohistochemistry}

After a survival time of 5 days, mice were deeply anesthetized, and the spinal cord and brain were removed and post-fixed in $4 \%$ paraformaldehyde-borate. Tissue sections were processed for GFP $(1: 1,000$; Molecular Probes, Eugene, OR) fluorescence immunohistochemistry according to standard protocols [29, 46]. For TPH IHC, the first antibody was sheep anti-TPH (1:2000; Chemicon International, Temecula, CA) and the second antibody included biotinylated donkey anti-sheep $\operatorname{IgG}(\mathrm{H}+\mathrm{L})$ (Lot no.68003, Jackson ImmunoResearch Lab., West Grove, PA) and streptavidin Alexa Fluor 350 conjugate (Lot no.49248A, S-11249, Invitrogen, Molecular Probes, Eugene, OR).

\section{Tissue analysis}

Immunofluorescence photomicroscope was visualized by using an Olympus IX81 photomicroscope. PRV-614-positive neurons were identified with red fluorescence, MC4R-GFP- expressing neurons were recognized by green fluorescence, and TPH-expressing neurons were recognized by blue fluorescence. The regions

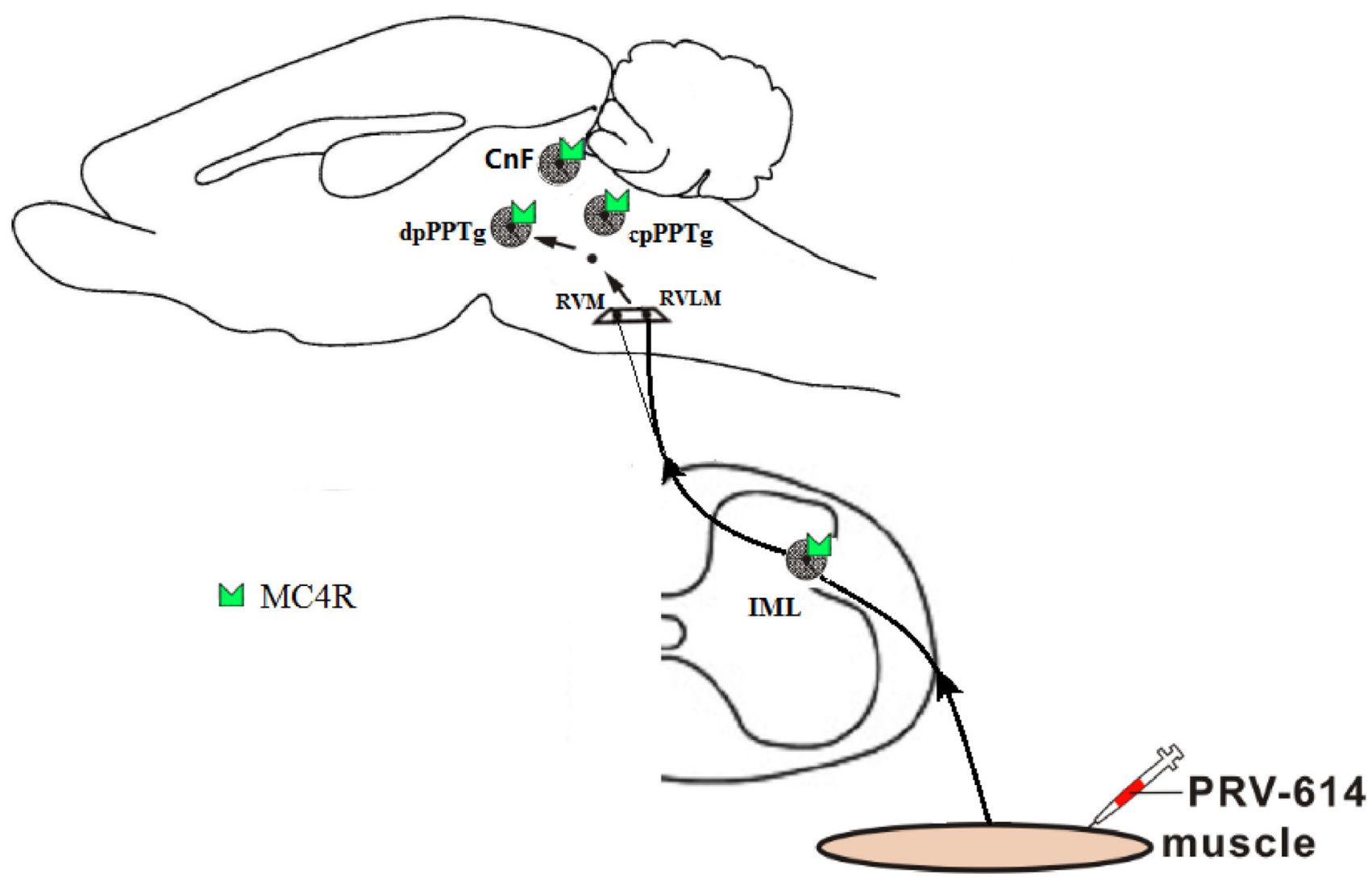

Figure 3: Summary diagram showed that the melanocortin-sympathetic pathway between the dpPPTg and skeletal muscle. Neurons within the dpPPTg send projections to the rostral ventral medulla (e.g., RVLM premotor neurons), which in turn projects to the sympathetic preganglionic neurons within the IML, which control skeletal muscle activity regulated by melanocortinsympathetic signals. PRV-614, pseudorabies virus-614; dpPPTg, the dissipated parts of PPTg; IML, intermediolateral column; RVLM, rostral ventrolateral medulla; RVMM, rostral ventromedial medulla. Some drawings were taken from HB Xiang (Brain 2013). 
in which positive cells were located were defined with reference to the atlases of Franklin KB and Paxinos G [47].

\section{ACKNOWLEDGMENTS AND FUNDING}

We gratefully acknowledge Dr. Lynn Enquist for providing us with PRV-614 and Dr. Joel Elmquist for providing MC4R-GFP transgenic mice. This work was partly supported by grants from National Natural Science Foundation of China (No. 81670240), and Special Fund of Fundamental Scientific Research Business Expense for Higher School of Central Government (2016CFB625), National Natural Science Foundation of Hubei Province (No. 2016CFB625, 2016CFB324), and Health and Family Planning Commission of Hubei Province (No. WJ2015MB008).

\section{CONFLICTS OF INTERESTS}

The authors have no conflicts of interest related to this paper.

\section{REFERENCES}

1. Zweig RM, Jankel WR, Hedreen JC, Mayeux R, Price DL. The pedunculopontine nucleus in Parkinson's disease. Annals of neurology. 1989; 26:41-46.

2. Meissner WG, Frasier M, Gasser T, Goetz CG, Lozano A, Piccini P, Obeso JA, Rascol O, Schapira A, Voon V, Weiner DM, Tison F, Bezard E. Priorities in Parkinson's disease research. Nature reviews Drug discovery. 2011; 10:377-393.

3. Rinne JO, Ma SY, Lee MS, Collan Y, Roytta M. Loss of cholinergic neurons in the pedunculopontine nucleus in Parkinson's disease is related to disability of the patients. Parkinsonism \& related disorders. 2008; 14:553-557.

4. Thevathasan W, Cole MH, Graepel CL, Hyam JA, Jenkinson N, Brittain JS, Coyne TJ, Silburn PA, Aziz TZ, Kerr G, Brown P. A spatiotemporal analysis of gait freezing and the impact of pedunculopontine nucleus stimulation. Brain. 2012; 135:1446-1454.

5. Thevathasan W, Pogosyan A, Hyam JA, Jenkinson N, Bogdanovic M, Coyne TJ, Silburn PA, Aziz TZ, Brown P. A block to pre-prepared movement in gait freezing, relieved by pedunculopontine nucleus stimulation. Brain. 2011; 134:2085-2095.

6. Thevathasan W, Pogosyan A, Hyam JA, Jenkinson N, Foltynie T, Limousin P, Bogdanovic M, Zrinzo L, Green AL, Aziz TZ, Brown P. Alpha oscillations in the pedunculopontine nucleus correlate with gait performance in Parkinsonism. Brain. 2012; 135:148-160.

7. Xiang HB, Zhu WZ, Guan XH, Ye DW. The cuneiform nucleus may be involved in the regulation of skeletal muscle tone by motor pathway: a virally mediated trans-synaptic tracing study in surgically sympathectomized mice. Brain. 2013; 136:e251-254.

8. Liu H, Kishi T, Roseberry AG, Cai X, Lee CE, Montez JM, Friedman JM, Elmquist JK. Transgenic mice expressing green fluorescent protein under the control of the melanocortin-4 receptor promoter. The Journal of Neuroscience. 2003; 23:7143-7154.

9. Kishi T, Aschkenasi CJ, Lee CE, Mountjoy KG, Saper $\mathrm{CB}$, Elmquist JK. Expression of melanocortin 4 receptor mRNA in the central nervous system of the rat. The Journal of Comparative Neurology. 2003; 457:213-235.

10. Alam M, Heissler HE, Schwabe K, Krauss JK. Deep brain stimulation of the pedunculopontine tegmental nucleus modulates neuronal hyperactivity and enhanced beta oscillatory activity of the subthalamic nucleus in the rat 6-hydroxydopamine model. Experimental Neurology. 2012; 233:233-242.

11. Alam M, Schwabe K, Krauss JK. The pedunculopontine nucleus area: critical evaluation of interspecies differences relevant for its use as a target for deep brain stimulation. Brain. 2011; 134:11-23.

12. Tanaka T, Masuzaki H, Yasue S, Ebihara K, Shiuchi T, Ishii $\mathrm{T}$, Arai $\mathrm{N}$, Hirata $\mathrm{M}$, Yamamoto $\mathrm{H}$, Hayashi $\mathrm{T}$, Hosoda K, Minokoshi Y, Nakao K. Central melanocortin signaling restores skeletal muscle AMP-activated protein kinase phosphorylation in mice fed a high-fat diet. Cell Metab. 2007; 5:395-402.

13. Elmquist JK. Hypothalamic pathways underlying the endocrine, autonomic, and behavioral effects of leptin. International Journal of Obesity and Related Metabolic Disorders. 2001; 25:S78-82.

14. Elmquist JK. Hypothalamic pathways underlying the endocrine, autonomic, and behavioral effects of leptin. Physiology \& Behavior. 2001; 74:703-708.

15. Xiang HB, Liu C, Guo QQ, Li RC, Ye DW. Deep brain stimulation of the pedunculopontine tegmental nucleus may influence renal function. Medical Hypotheses. 2011; 77: $1135-1138$.

16. Lee TK, Lois JH, Troupe JH, Wilson TD, Yates BJ. Transneuronal tracing of neural pathways that regulate hindlimb muscle blood flow. American journal of physiologyregulatory integrative and comparative physiology. 2007; 292:R1532-1541.

17. Zechner JF, Mirshahi UL, Satapati S, Berglund ED, Rossi J, Scott MM, Still CD, Gerhard GS, Burgess SC, Mirshahi T, Aguirre V. Weight-independent effects of roux-en-Y gastric bypass on glucose homeostasis via melanocortin-4 receptors in mice and humans. Gastroenterology. 2013; 144:580-590 e587.

18. Liu TT, Liu BW, He ZG, Feng L, Liu SG, Xiang HB. Delineation of the central melanocortin circuitry controlling the kidneys by a virally mediated transsynaptic tracing study in transgenic mouse model. Oncotarget. 2016; 7:69256-69266. doi: 10.18632/oncotarget.11956. 
19. Sohn JW, Harris LE, Berglund ED, Liu T, Vong L, Lowell BB, Balthasar N, Williams KW, Elmquist JK. Melanocortin 4 receptors reciprocally regulate sympathetic and parasympathetic preganglionic neurons. Cell. 2013; 152:612-619.

20. Rossi J, Balthasar N, Olson D, Scott M, Berglund E, Lee CE, Choi MJ, Lauzon D, Lowell BB, Elmquist JK. Melanocortin-4 receptors expressed by cholinergic neurons regulate energy balance and glucose homeostasis. Cell Metabolism. 2011; 13:195-204.

21. Hao Y, Guan XH, Liu TT, He ZG, Xiang HB. Hypothesis: The central medial amygdala may be implicated in sudden unexpected death in epilepsy by melanocortinergicsympathetic signaling. Epilepsy \& behavior. 2014; 41C: 30-32.

22. Hao Y, Liu TT, He ZG, Wu W, Xiang HB. Hypothesis: CeM-PAG GABAergic circuits may be implicated in sudden unexpected death in epilepsy by melanocortinergic signaling. Epilepsy \& behavior. 2015; 50:25-28.

23. Liu TT, He ZG, Tian XB, Liu C, Xiang HB, Zhang JG. Hypothesis: Astrocytes in the central medial amygdala may be implicated in sudden unexpected death in epilepsy by melanocortinergic signaling. Epilepsy \& behavior. 2015; 42:41-43.

24. Song YT, Liu TT, Feng L, Zhang T, Xiang HB. Melanocortin-4 receptor expression in the cuneiform nucleus is involved in modulation of opioidergic signaling. Journal of Huazhong University of Science and Technology Medical sciences. 2015; 35:662-665.

25. Xu AJ, Liu TT, He ZG, Wu W, Xiang HB. CeA-NPO circuits and REM sleep dysfunction in drug-refractory epilepsy. Epilepsy \& behavior. 2015; 51:273-276.

26. Xu LJ, Liu TT, He ZG, Hong QX, Xiang HB. Hypothesis: CeM-RVLM circuits may be implicated in sudden unexpected death in epilepsy by melanocortinergic-sympathetic signaling. Epilepsy \& behavior. 2015; 45:124-127.

27. Xu AJ, Liu TT, He ZG, Hong QX, Xiang HB. STN-PPTg circuits and REM sleep dysfunction in drug-refractory epilepsy. Epilepsy \& behavior. 2015; 51:277-280.

28. Xiang HB, Zhu WZ, Guan XH, Ye DW. Possible mechanism of deep brain stimulation for pedunculopontine nucleus-induced urinary incontinence: a virally mediated transsynaptic tracing study in a transgenic mouse model. Acta neurochirurgica. 2013; 155:1667-1669.

29. Ye D, Guo Q, Feng J, Liu C, Yang H, Gao F, Zhou W, Zhou L, Xiang H, Li R. Laterodorsal tegmentum and pedunculopontine tegmental nucleus circuits regulate renal functions: Neuroanatomical evidence in mice models. Journal of Huazhong University of Science and Technology Medical sciences. 2012; 32:216-220.

30. Stanley S, Pinto S, Segal J, Perez CA, Viale A, DeFalco J, Cai X, Heisler LK, Friedman JM. Identification of neuronal subpopulations that project from hypothalamus to both liver and adipose tissue polysynaptically. Proceedings of the National Academy of Sciences of the United States of America. 2010; 107:7024-7029.

31. Chen M, He ZG, Liu BW, Li ZX, Liu SG, Xiang HB. Parafascicular nucleus-heart neural crosstalk: Implications for seizure-induced myocardial stunning. Epilepsy \& behavior. 2016; 63:135-137.

32. Chen M, He ZG, Liu SG, Xiang HB. Motor cortexperiaqueductal gray-rostral ventromedial medulla neuronal circuitry may involve in modulation of nociception by melanocortinergic-opioidergic signaling. International journal of clinical and experimental pathology. 2016; 9:7897-7907.

33. Feng MH, He ZG, Liu BW, Li ZX, Wu DZ, Liu SG, Xiang HB. Parafascicular nucleus circuits: implications for the alteration of gastrointestinal functions during epileptogenesis. Epilepsy \& Behavior. 2016; 64:295-298.

34. Liu BW, He ZG, Shen SE, Xiang HB. CeA-RVMM serotonergic circuits and sudden unexpected death in epilepsy. International journal of clinical and experimental medicine. 2016; 9:9752-9758.

35. Liu BW, Liu QQ, Liu SG, Xiang HB. Renal disease and neural circuits: brain-kidney crosstalk. International journal of clinical and experimental medicine. 2016; 9: 5326-5333.

36. He ZG, Zhang DY, Liu SG, Feng L, Feng MH, Xiang HB. Neural circuits of pain and itch processing involved in anterior cingulate cortex. International journal of clinical and experimental medicine. 2016; 9:22976-22984.

37. Zhou Y, He Z, Liu T, Feng M, Zhang D, Xiang H. The neuroanatomical circuitry between kidney and rostral elements of brain: a virally mediated transsynaptic tracing study in the mouse. Journal of Huazhong University of Science and Technology Medical sciences. 2017; 37:63-69.

38. Feng M, He Z, Liu B, Li Z, Tao G, Wu D, Xiang H. Consciousness loss during epileptogenesis: implication for VLPO-PnO circuits. International journal of physiology, pathophysiology and pharmacology. 2017; 7:152-157.

39. Liu BW, Liu QQ, Liu SG, Xiang HB. Renal disease and neural circuits: brain-kidney crosstalk. International journal of clinical and experimental medicine. 2016; 9:5326-5333.

40. Karachi C, Grabli D, Bernard FA, Tande D, Wattiez N, Belaid H, Bardinet E, Prigent A, Nothacker HP, Hunot S, Hartmann A, Lehericy S, Hirsch EC, et al. Cholinergic mesencephalic neurons are involved in gait and postural disorders in Parkinson disease. The Journal of Clinical Investigation. 2010; 120:2745-2754.

41. Rotto-Percelay DM, Wheeler JG, Osorio FA, Platt KB, Loewy AD. Transneuronal labeling of spinal interneurons and sympathetic preganglionic neurons after pseudorabies virus injections in the rat medial gastrocnemius muscle. Brain research. 1992; 574:291-306.

42. Kerman IA, Enquist LW, Watson SJ, Yates BJ. Brainstem substrates of sympatho-motor circuitry identified using trans-synaptic tracing with pseudorabies virus recombinants. The Journal of Neuroscience. 2003; 23:4657-4666. 
43. Shafei MN, Nasimi A, Alaei H, Pourshanazari AA, Hosseini M. Role of cuneiform nucleus in regulation of sympathetic vasomotor tone in rats. Pathophysiology. 2012; 19:151-155.

44. Takakusaki K, Habaguchi T, Ohtinata-Sugimoto J, Saitoh K, Sakamoto T. Basal ganglia efferents to the brainstem centers controlling postural muscle tone and locomotion: a new concept for understanding motor disorders in basal ganglia dysfunction. Neuroscience. 2003; 119:293-308.

45. Campos RR, Carillo BA, Oliveira-Sales EB, Silva AM, Silva NF, Futuro Neto HA, Bergamaschi CT. Role of the caudal pressor area in the regulation of sympathetic vasomotor tone. Brazilian Journal of Medical and Biological Research. 2008; 41:557-562.

46. Pan XC, Song YT, Liu C, Xiang HB, Lu CJ. Melanocortin-4 receptor expression in the rostral ventromedial medulla involved in modulation of nociception in transgenic mice. Journal of Huazhong University of Science and Technology Medical sciences. 2013; 33:195-198.

47. Franklin KB, Paxinos G. The mouse Brain in Stereotaxic Coordinates. Third Edition. San Diego, CA: Academic Press. 2007. 